Paper:

\title{
MDVM System Concept, Paging Latency and Round-2 Randomized Leader Election Algorithm in SG
}

\author{
Susmit Bagchi*, Hafizur Rahaman**, and Purnendu Das ${ }^{* * *}$ \\ *Deptt. of Information Technology, Sikkim Manipal Inst. of Technology \\ Majitar, Sikkim, India \\ E-mail: susmit-bagchi@lycos.com \\ ${ }^{* *}$ Deptt. of Information Technology, Bengal Engineering and Science University \\ Shibpur, Howrah, India \\ E-mail: rahaman_h@hotmail.com \\ ${ }^{* * *}$ School of Information Technology, Bengal Engineering and Science University \\ Shibpur, Howrah, India \\ [Received October 28, 2005; accepted April 17, 2006]
}

The future trend in the computing paradigm is marked by mobile computing based on mobileclient/server architecture connected by wireless communication network. However, the mobile computing systems have limitations because of the resourcethin mobile clients operating on battery power. The MDVM system allows the mobile clients to utilize memory and CPU resources of Server-Groups (SG) to overcome the resource limitations of clients in order to support the high-end mobile applications such as, mcommerce and virtual organization (VO). In this paper the concept of MDVM system and the architecture of cellular network containing the SG are discussed. A round-2 randomized distributed algorithm is proposed to elect a unique leader and co-leader of the SG. The algorithm is free from any assumption about network topology, buffer space limitations and is based on dynamically elected coordinators eliminating single point of failure. The algorithm is implemented in distributed system setup and the network-paging latency values of wired and wireless networks are measured experimentally. The experimental results demonstrate that in most cases the algorithm successfully terminates in first round and the possibility of second round execution decreases significantly with the increase in the size of SG $\left(\left|N_{a}\right|\right)$. The overall message complexity of the algorithm is $O\left(\left|N_{a}\right|\right)$. The comparative study of network-paging latencies indicates that $3 \mathrm{G} / 4 \mathrm{G}$ mobile communication systems would support the realization of MDVM system.

Keywords: GPRS, GSM, mobile computing, network paging latency, leader election algorithms

\section{Introduction}

The availability of portable computing devices having access to WWW, such as smart phones and PDAs, has created a set of high-end mobile applications such as, mcommerce, SES [21] and virtual organization [22]. The mobile devices are limited in hardware resources [24], battery power and operate in doze mode to reduce power requirement [23]. The existing wireless communication technology is restricted in terms of bandwidth and reliability [23]. The existing general-purpose operating systems offer very little support for managing and adapting to the mobile computation paradigm [25]. As a novel approach, the concept of Mobile Distributed Virtual Memory (MDVM) is introduced to enable mobile clients exploiting server resources using the mobile communication network [20]. The Server-Group (SG) of MDVM system allows mobile clients to utilize the server CPU and memory for the data cache and process execution purposes [20]. In this paper, we describe the MDVM system concept, the architecture of mobile communication system consisting of Server-Group (SG) and a randomized distributed algorithm for electing a unique leader and co-leader of the SG in at most two election rounds. The distinguishing features of the proposed algorithm are as followings:

- The proposed distributed randomized algorithm does not assume any statically assigned processor or server IDs. The algorithm assigns the unique IDs to the MDVM servers online.

- The server IDs may not be strictly monotonically increasing or decreasing according to the sequence of registration of the MDVM servers in a SG.

- The algorithm takes at most two rounds to elect a unique leader and co-leader. The experimental results indicate that the algorithm terminates in first round in majority cases and the possibility of entering in the second round of execution decreases significantly with the increase in the size of SG.

- While executing in the second round, the algorithm offers high filter ratio values indicating that the number of MDVM servers participating in the second 
round election phase is substantially reduced as compared to the first round of execution. This reduces the network message complexity further.

- The overall message complexity of the algorithm is considerably low and is in the order of the size of $\mathrm{SG}, \mathrm{O}\left(\left|N_{a}\right|\right)$.

- The election rounds are supervised by different coordinators in two different rounds. The coordinator of the second round is elected dynamically. This reduces the possibility of single point of failure.

- Unlike the other leader election algorithms, the proposed algorithm is free from any assumptions such as, static node values, network topology and buffer sizes etc.

- Unlike the probabilistic leader election algorithms proposed earlier, the proposed algorithm does not require several rounds of simultaneous multicasts on the completion of leader and co-leader election in a SG.

We have implemented the algorithm in distributed system setup and have measured the network-paging latency values between a mobile-client and a server in SG. We have compared the paging-latency values for various page sizes in 100Mbps LAN, 10Mbps Wireless-VPN and 2.5G GPRS systems. The experimental results demonstrate that the algorithm terminates in first round in majority cases by electing a unique leader and co-leader however, in few cases the algorithm enters in the second round election phase. The round ratio and filter ratio of the algorithm decrease significantly with the increase in the size of SG. The rest of the paper is organized as followings. Section 2 describes the related work. Section 3 describes the high-end mobile applications along with the technological trends and its limitations. Section 4 illustrates the MDVM system concept and the architecture of mobile communication system containing the SG. Section 5 and section 6 describe the leader election algorithm and the experimental results respectively. Section 7 concludes the paper.

\section{Related Works}

The leader election algorithms are widely studied in the context of wired and wireless networks. The concept of MDVM system uses the Server-Group (SG) placed in the mobile communication architecture [20]. The SG is consisting of a number of MDVM servers connected by wired TCP/IP network offering resources to the mobile clients. Researchers have proposed a leader election algorithm in a synchronous ring of $n$ servers having predetermined unique identifiers $[8,10]$. The algorithm uses $\mathrm{O}(n)$ messages if the identifiers are chosen from some countable set. In the worst case, the message complexity of the algorithm is $\mathrm{O}(n \log n)$ [10]. It is reported that the deterministic algorithms for the leader election in bidirectional rings $[15,17,18]$ and unidirectional rings have $\mathrm{O}(n \log n)$ message complexity [10]. These deterministic algorithms work in either synchronous or asynchronous communication models. Although it is shown that the asynchronous communication system leads to $\mathrm{O}(n \log n)$ message complexity as a lower bound [17], however, the proof may not be applicable to the synchronous communication model [10]. The one of the difficulties of leader election algorithm proposed in [10] is that it may require a very large number of synchronous rounds in the worst case. In addition, the algorithm assumes statically assigned unique integer IDs to the processors. One of the restrictions of the algorithm is that it discriminates the wake-up-processes from the awakened-processes [10]. Researchers have proposed a self-stabilizing algorithm for leader election in a tree graph [1]. It is illustrated that it may not be possible to design a self-stabilizing algorithm for a tree with the possibility of electing a leaf node as the leader. The proposed algorithm is dependent on the structure of the tree and assumes a predetermined fixed number of nodes in a tree. Another difficulty of the algorithm is that it is dependent on a central daemon and the multiple privileged nodes of the tree should not be adjacent [1]. However, the algorithm does not assume any particular order while choosing a set of active nodes [1]. Another deterministic and self-stabilizing algorithm is proposed in [7] to elect a leader in a ring based on a central daemon. However, the algorithm uses constant space and is self-stabilizing in $\mathrm{O}\left(n^{2}\right)$ steps [7]. The algorithm generates a large number of tokens, which increases the complexity of the algorithm considerably. The non-randomized leader election algorithms for the failure-prone asynchronous network can be classified as: Gallager-Humblet-Spira type algorithms, logical network partition based algorithms and the algorithms assuming reliable message delivery [6]. On the other hand, the probabilistic leader election algorithms are generally classified as the randomized algorithms $[6,19]$. In general, the message complexity of the probabilistic algorithms varies linearly with the group size and the algorithms require several rounds of $\mathrm{O}(N)$ simultaneous multicasts [6]. Hence, this class of probabilistic algorithms may deteriorate the delivery performance of the underlying network. Researchers have proposed a probabilistic leader election algorithm for large groups based on the practical settings [6]. The algorithm offers scalability and assumes week membership in an unreliable network. However, the algorithm is fairly complex and message complexity could be very high depending on the filter value $K$ [6]. In addition, as the view of a process in the group may not be identical for all members of the group, the multiplicity of multicast message rounds may enhance the consumption of network bandwidth considerably, duplicate messaging and may lead to the requirement of message ordering. There exists possibility that $N_{i}$ value [6] seen by different members may not be consistent and may require multiple circulations. In another approach, a space optimal self-stabilizing leader election protocol for anonymous unidirectional ring is proposed [11]. The algorithm has $\mathrm{O}\left(m_{N}\right)$ space complexity [11]. One of the difficulties of the algorithm is its dependence 
Table 1. 3G and 4G mobile communication technology capabilities.

\begin{tabular}{|l|l|l|}
\hline Technology & $3^{\text {rd }}$ Generation $(3 \mathrm{G})$ & $4^{\text {th }}$ Generation $(4 \mathrm{G})$ \\
\hline Speed & $384 \mathrm{Kbps}-2 \mathrm{Mbps}$ & $100 \mathrm{Mbps}$ \\
\hline Applications & $\begin{array}{l}\text { Voice, email, video-on-demand, } \\
\text { multimedia }\end{array}$ & $\begin{array}{l}\text { Voice, high-speed internet access, } \\
\text { high-resolution video, IPv6 }\end{array}$ \\
\hline
\end{tabular}

on a probabilistic scheduler, which may not be realizable. Apart from the wired network, a set of leader election algorithms is proposed for mobile ad-hoc networks [2, 4, 5] and wireless sensor networks [3]. In another approach towards the leader election algorithm in distributed systems having quantum resources, an algorithm is proposed in the framework of quantum computing [9]. The proposed solution is highly theoretical in nature and may not be realizable at present.

\section{High-End Mobile Applications}

The mobile devices equipped with $3 \mathrm{G}, 4 \mathrm{G}$ and higher mobile communication technologies have created the possibility to realize a set of high-end mobile applications such as, (1) Mobile enterprise applications in business environments (examples are m-commerce and SES) [21, 22], (2) Virtual organization based on completely mobile working spaces (an example is MOWAHS) [22] and (3) Mobile distributed computing where the cheap and resource-thin mobile devices can run mobile applications on remote servers in a distributed manner using RMI/RPC technology. A set of typical activities performed by the mobile enterprise applications in a virtual office environment can be summarized as [21, 22, 28]: (1) Accessing documents and querying remote databases, (2) Data mailing / messaging between mobile processes, (3) Invoking remote methods on servers (RMI, RPC etc.) and (4) Data exchange between mobile clients and stationary server processes. Although the mobile communication technology is improving rapidly, however, the resource limitations of battery operated mobile devices are limiting the nature of mobile applications.

\subsection{Technological Trends and Limits}

The trend in the modern computer industry is to produce mobile computers [27]. Due to rapid development in hardware technology, the mobile computers are getting equipped with powerful microprocessors, high-speed network capabilities and other resources. The next generation mobile communication technology will be $3 \mathrm{G}$ and $4 \mathrm{G}$ as proposed in industry such as NTT DoCoMo offering significantly higher wireless communication bandwidth and reliability as compared to $2.5 \mathrm{G}$ [21]. The proposed capabilities of $3 \mathrm{G}$ and $4 \mathrm{G}$ mobile communication technologies are described in Table 1 [20]. A set of restrictions that would exist in future is: (1) Limitation of computing time and peripheral devices due to power consumption [28], (2) Unavailability of secondary storage as a peripheral of mobile devices, (3) Performance limitation of FLASH memory, (4) Physical specifications of mobile devices are to be lightweight and small in size, and (5) The cost of wireless communication bandwidth is high. The MDVM concept extends the server resources to mobile clients using mobile communication interface to reduce the resource constraints of mobile devices.

\section{The Concept and Architecture of MDVM System}

The concept of Mobile Distributed Virtual Memory (MDVM) will allow a roaming employee of the virtual organization to utilize the resources of remote servers. The MDVM design concept extends the CPU and memory resources of servers to the mobile clients using a wireless communication network interface. The mobile clients can use server memory and CPU resources to achieve functionalities such as, memory space for large data cache, virtual memory space and CPU for process execution. The data cache availability can be improved within the framework of the MDVM design employing data replication and cache consistency mechanisms. The conceptual view of the MDVM design architecture is illustrated in Fig.1. From the view of a mobile client, MDVM is the remote virtual memory space accessible from anywhere in the mobile communication network, and can migrate from cell to cell in order to save communication bandwidth, time and cost. The $4 \mathrm{G}$ mobile communications standard supports inter-domain protocol conversion. The W-CDMA [21] based mobile network can be used to connect to a TCP/IP based corporate LAN, web servers and the MDVM servers.

\subsection{Dynamic Server-Group}

The MDVM design concept considers a group of servers connected by high-speed wired network residing within the cells. The servers offer MDVM as a service to mobile clients through the wireless network. The MDVM servers form a server-group (SG) as shown in Fig.2. Individual servers in a $\mathrm{SG}$ serve the mobile clients in the corresponding cell. The members of a SG elect a leader and co-leader to perform the periodic tasks in a SG. Such tasks are comprised of keeping the overall status of the SG-members, resource allocation history and to keep the replica of storage segments of MDVM located on other members of SG as a fault tolerance mechanism. The coleader assumes the responsibility of the leader if the leader 


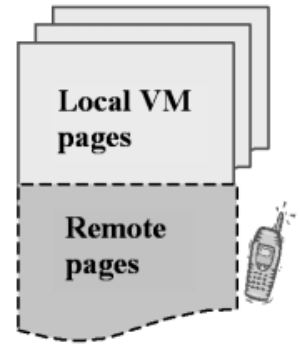

Mobile Client

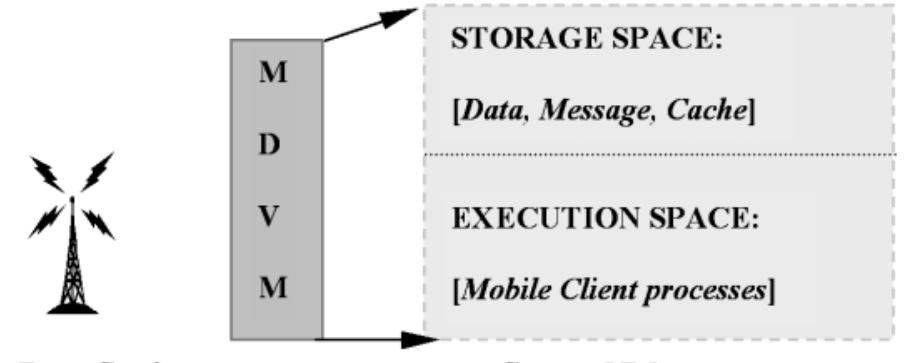

Base Station

\section{Server VM}

Fig. 1. MDVM for mobile clients.

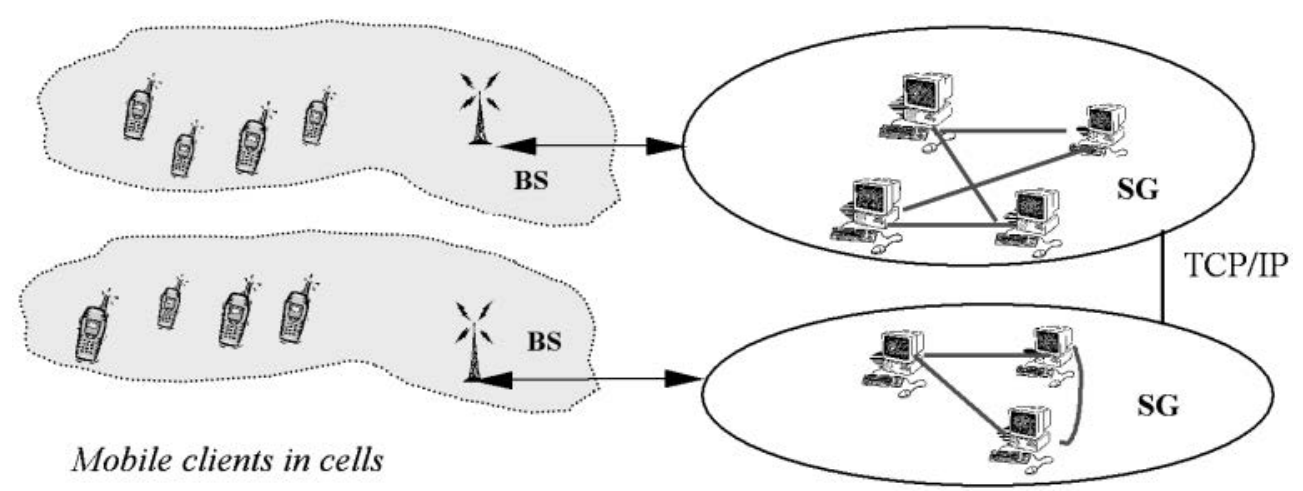

Fig. 2. The server groups and mobile clients.

crashes. This eliminates the necessity of "stop and leaderreelection" in the system at any point of time due to leader crash. On the other hand, the co-leader shares the load on a leader eliminating the problem of central point of load concentration along with the saturation of network bandwidth around the leader. The members of a SG are required to handle the memory migration from another SG requiring inter-SG communication system. The $4 \mathrm{G}$ mobile networks standard supports protocol conversion techniques. This enables the two SGs to communicate using TCP/IP protocol stack over the high bandwidth wired link.

\subsection{SG in Cellular Network}

The architecture of mobile communication network containing MDVM servers is shown in Fig.3. The system architecture is consisting of mobile clients (MC) and stationary servers. The stationary servers of SG are placed in the Digital Mobile Communication Network (DMCN) on the TCP/IP bus. According to the IMT-2000 standard [21] the DMCN has capability to support TCP/IP network stack and protocol conversions. The servers in SG are interconnected by high-speed wired TCP/IP network and reside in between i-Gateway and PGWM. The PGWM interfaces TCP/IP bus and NBB. The job of NBB is to support the basic cellular communication system backbone. The data packet processing in the mobile communication system is handled by PP and MLSC. The BS in a cell is connected to NBB via MLSC. In 3G and higher mobile communication standard, the DMCN has capabil- ity to interface the Internet servers using special server i-Gateways using TCP/IP protocol. Hence, the MCs may communicate to the MDVM servers, corporate LAN and web servers via DMCN using the TCP/IP interface controlled by PGWM. Similarly, the servers in SG can reach corporate LAN and Internet via i-Gateway interface. The inter-SG communication is based on TCP/IP interface controlled by PGWM.

\section{The Leader Election Algorithm}

Initially, the SG in a cell assigns a coordinator temporarily. The job of the coordinator is to initiate the round one election process and to delegate the intermediate results to all the other participating servers through multicast. On reception of the intermediate results, individual MDVM servers execute the distributed randomized algorithm to elect a leader and a co-leader. This computation phase does not involve any message communication over the network. Once the round one election phase is over, the servers will come to a state indicating whether the round one election is enough to decide a leader and co-leader uniquely or not. If a leader and co-leader are elected with uniqueness, then the algorithm terminates. It should be noted that the algorithm does not require simultaneous multicasting the election outcome to the other servers, as each MDVM server executing the algorithm in distributed fashion would converge to a unique solution. This reduces the overall message complexity by eliminating several rounds of simultaneous multicasting. In an- 


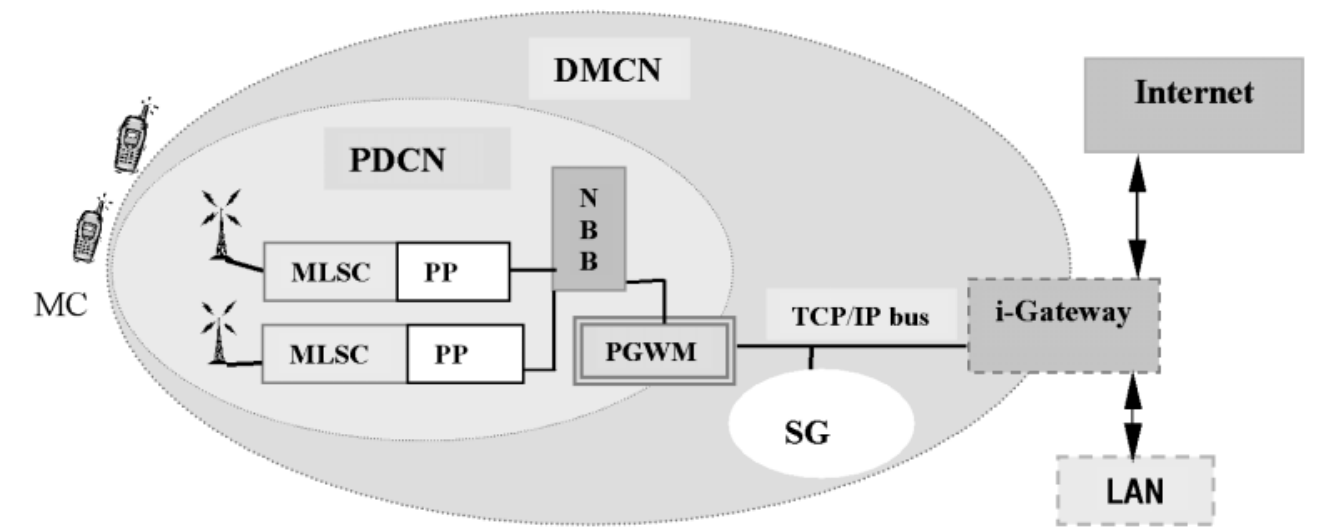

PDCN: Packet Data Communication Network, MLSC: Mobile Local Switch Center, PP: Packet Processor, NBB: Network Backbone, PGWM: Packet Gateway Module.

Fig. 3. The SG embedded into cellular network.

other case, if the individual servers are unable to determine a unique leader and co-leader randomly in round one, then the algorithm enters into the second round of election process. However, there are three distinctive features of the algorithm as mentioned below:

- The coordinator for the second round election may be different from the coordinator of the first round election process. The selection of the second coordinator is random based on the outcome of the algorithm in first round. This reduces the possibility of having the single point of failure due to the failure of coordinator.

- The number of MDVM servers entering into round two election phase are considerably lower as compared to the number of servers participated in the first round election process. This reduces the network message complexity significantly.

- The algorithm terminates in round two by randomly electing the unique leader and co-leader of a SG.

The entire algorithm is presented in three parts such as, registration phase, randomization phase and distributed election phase for the easiness of understanding and presentation. In the following sections, the description of the three parts of the algorithm is described and the pseudo code of the algorithm is given in $\mathrm{C}$ language syntax for easy understanding.

\subsection{The Registration Phase}

In this phase, the individual nodes, those are interested to be the MDVM servers by offering resources to the mobile clients, register the individual IP addresses to the coordinator. The coordinator acts as a multithreaded server carrying out the IP address registration process for all the MDVM servers. The coordinator calculates the network latency values (nw_latency) by measuring the time interval between the events of establishment of the network connection (between the coordinator and a server) and the reception of the registration message packet sent by the MDVM server (<"Register",ip >). As the nw_latency values are dependent on the time-varying network conditions, congestion states and the time delays introduced by multithreaded coordinator due to the thread scheduling delays, the nw_latency values are unpredictable and prone to be randomly varying for the MDVM servers. The nw_latency value of the coordinator is calculated by averaging such values of all the registered MDVM servers. The coordinator of the first round election process works on the basis of the timeout interval. The coordinator registers the MDVM servers who respond within the timeout interval. The timeout interval is kept sufficiently long. In our experiments, we have assigned the timeout interval as thirty minutes. On the event of timeout, the coordinator multicasts the ordered tuple containing IP addresses, nw_latency values and IDs to the registered MDVM servers (<ip, nw_latency, ID>). The node IDs are assigned online by the coordinator based on the nw_latency values and the sequence of registration represented by the index values. Suppose, $N_{a}$ be a set of MDVM servers registered to the coordinator and $I_{d}$ is a set of unique IDs. Then, $I_{d}=B_{1} \cup B_{2} \cup B_{3}$ and $\left|N_{a}\right|>0$ where, $B_{1}=\left\{\alpha: \alpha \in I^{+}, 0<\alpha<2\left|N_{a}\right|\right\}$, $B_{2}=\left\{2\left|N_{a}\right|\right\}$ and, $B_{3}=\left\{\beta: \beta \in I^{+}, \beta>2\left|N_{a}\right|\right\}$. Let, the registered server set is depicted as the set of ordered pair $A=\left\{\left\langle n_{i}, k_{i}\right\rangle: i \in I^{+}, i>0, n_{i} \in N_{a}, k_{i} \in I_{d}\right\}$. The algorithm assigns the IDs to the individual registered MDVM servers such that, if $\left\langle n_{i}, k_{i}\right\rangle,\left\langle n_{j}, k_{j}\right\rangle \in A$ and $i<j$, then $\left(k_{i}<k_{j}\right) \vee\left(k_{i}>k_{j}\right) \vee\left(k_{i}=k_{j}\right)$. Hence, the assigned IDs may not be strictly sequentially increasing or decreasing according to the sequentially increasing or decreasing index values. The pseudo code of the registration and unique ID allocation phase of the algorithm is given in Fig.4 in $\mathrm{C}$ language syntax.

\subsection{The Randomization Phase}

On reception of the multicast message from the coordinator, the individual MDVM server in $N_{a}$ initiates randomization phase locally. Every MDVM servers in 


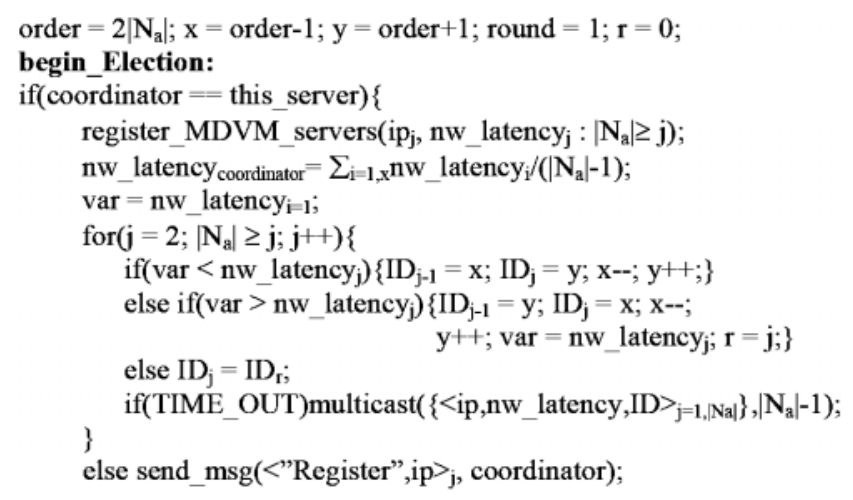

Fig. 4. Registration and ID assignment phase of the algorithm.

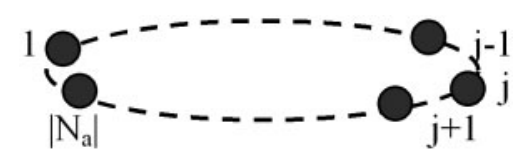

Fig. 5. Logical ring of indexed MDVM servers.

$N_{a}$ are indexed by $j$ and are assigned to two random variables namely, rand_index and ring_id. The indexed MDVM servers in $N_{a}$ form a logical ring as illustrated in Fig.5. The ring id values of all registered MDVM servers in the ring are computed by adding the IDs of the left and right neighbours of a server in the ring along with its own ID. The pseudo code of the algorithm illustrating the ring id calculation is given in $\mathrm{C}$ language syntax in Fig.6. There exists a set of methods to generate random and pseudo-random numbers; however, the linear congruence method (LCM) is the simplest procedure [26]. The randomization function applied to design the proposed algorithm is inspired by LCM because it is simple, computationally inexpensive and provides acceptable performance limits while generating a set of pseudo-random numbers. The randomization function applied by the algorithm is a recurrence relation given as, $t_{i+1, j}=\left(\left(\partial_{j}-i+1\right) \cdot\left|N_{a}\right| \cdot\left(t_{i, j}-\left\lfloor t_{i, j}\right\rfloor\right)^{-1}+1\right) \cdot \gamma^{-1}$ where, $t_{0, j}=$ nw_latency $_{j}$ for all $n_{j} \in N_{a}, t_{i, j} \in R^{+}-Q^{+}$where $R^{+}$and $Q^{+}$are set of positive real and positive rational numbers respectively, $\gamma=\left\lfloor\left(\sum_{\left.k=1, \mid N_{a}\right\rfloor} \mathrm{ID}_{k}\right)^{0.5}\right\rfloor$ and $\partial_{j}=$ $\sum_{z=j-1, j+1} \mathrm{ID}_{z}, i=0,1,2, \ldots \partial_{j}-1$. The rand index values of all MDVM servers are computed by applying the recurrence relation based on the ring id, nw latency and the ID values of individual MDVM servers as illustrated in Fig.6. On the completion of computation of rand index values, the MDVM servers enter into the distributed election phase of the algorithm.

\subsection{The Distributed Election Phase}

In this terminal phase of the algorithm, each MDVM server in $N_{a}$ computes the set of nodes $\left(S_{l} \subset N_{a}\right)$ having minimum rand_index value. The outcome may fall into three classes, such as, (1) $\left|S_{l}\right|$ is unity, (2) $\left|S_{l}\right|>2$ and (3) $\left|S_{l}\right|=2$. In the first case, the leader election algo-

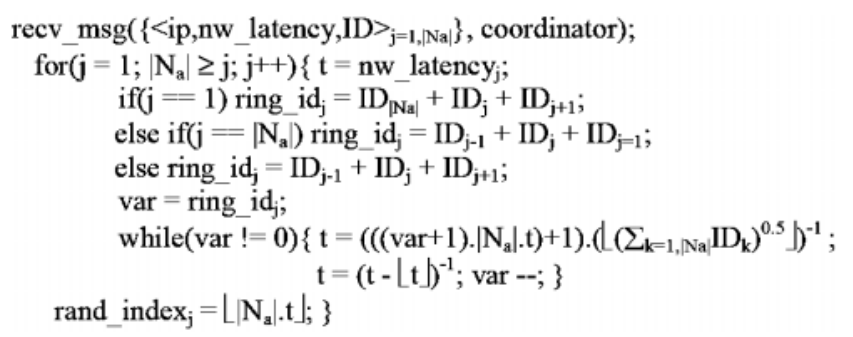

Fig. 6. Randomization phase of the algorithm.

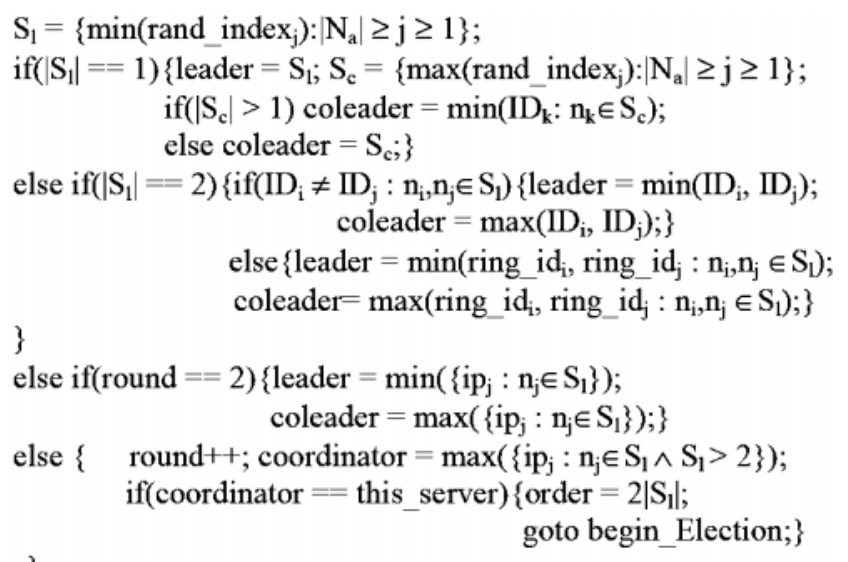
\}

Fig. 7. Distributed election phase of the algorithm.

rithm terminates and the member of $S_{l}$ is elected as leader. Accordingly, a set of nodes $\left(S_{c} \subset N_{a}\right)$ having maximum rand index value is computed. If, $\left|S_{c}\right|>1$, the member of $S_{c}$ having minimum ID value is elected as the co-leader. Otherwise, if $\left|S_{c}\right|=1$, then this unique member is elected as the co-leader. However, if $\left|S_{l}\right|=2$, then the algorithm terminates in round one by electing the member of this set as the leader having the minimum ID value. The other member of $S_{l}$ is elected as the co-leader in this case. On the contrary, if the ID values are identical, the algorithm elects the leader and co-leader based on the minimum and maximum values of ring id of the two MDVM servers respectively. Otherwise, if $\left|S_{l}\right|>2$, the algorithm enters in the second round of election process and a new coordinator is elected from the set $S_{l}$ having maximum IP address. This newly elected coordinator hold the round two election process and the number of MDVM servers participating in the second round election process is equal to $\left|S_{l}\right|$ $\left(2<\left|S_{l}\right|<\left|N_{a}\right|\right)$. In the worst case, if the algorithm fails to elect a unique leader and co-leader from $S_{l}\left(2 \leq\left|S_{l}\right|\right.$ $<\left|N_{a}\right|$ and round $\left.=2\right)$ in the second round due to the equality of two servers in all respect, the tie is broken by electing the leader having minimum IP address and the co-leader having the maximum IP address. The pseudo code representation of the distributed election phase of the algorithm is given in $\mathrm{C}$ language syntax in Fig.7. 
Table 2. Experimental results.

\begin{tabular}{|l|l|l|}
\hline & Round ratio & Filter ratio \\
\hline Experiment-I & 0.428 & 0.6 \\
\hline Experiment-II & 0.25 & 0.3 \\
\hline Experiment-III & 0.12 & 0.2 \\
\hline Experiment-IV & 0.5 & $0.0267,0.034,0.0467,0.0134,0.06$ \\
\hline
\end{tabular}

\section{Experimental Results}

The algorithm is implemented in the PC based distributed system environment. The realization of the algorithm is comprised of Windows socket based application modules written in $\mathrm{C}$ language implementing the clientserver computing model. Initially, a server is configured to act as the temporary coordinator holding the first round of election process and later, the algorithm elects another coordinator in case the algorithm enters in the second round. The experiments are carried out based on four different sets of MDVM servers having different number of servers in each set. The servers in SG are connected by $10 \mathrm{Mbps}$ Ethernet wired LAN. The network-paging latency values are measured through page-sized data IO between a client and a server connected by LAN, Wireless VPN and GPRS system. The sets of experiments are as followings: [1] Experiment - I: In this case the total number of MDVM servers in the leader election algorithm is equal to 5, [2] Experiment - II: In this case the number of servers in a SG is equal to 10, [3] Experiment III: In this case the total number of MDVM servers executing the algorithm is equal to 15 and [4] Experiment IV: In this experiment, a large distributed system setup is emulated by implementing ten different instances of the algorithm on fifteen server machines creating a large distributed system comprised of 150 MDVM servers. However, it should be noted, this emulated large distributed system tries to understand the performance of the algorithm by measuring the number of times the algorithm goes into the second round of election process. Each of the four sets of experiments is carried out ten times each in order to understand the random behaviour of the algorithm. [5] Experiment - V: Measurement of roundtrip network-paging latency between a client and a server connected by $100 \mathrm{Mbps}$ LAN, 10Mbps Wireless VPN and 2.5G GPRS system for $4 \mathrm{~KB}, 16 \mathrm{~KB}$ and $32 \mathrm{~KB}$ page sizes. The experimental results are given in Table 2 . The round ratio (round2/round1) illustrates the behaviour of the algorithm by measuring the total number of times it requires second round of election in ten experiment instances for the increasing number of MDVM servers. Accordingly, the filter ratio illustrates the performance of the algorithm by measuring the ratio of the number of servers entering in the second round of election process to the initial total number of MDVM servers in first round. The performance of the algorithm is given in Fig.8 and Fig.9 illustrating the variation of the round ratio and filter ratio respectively, where the horizontal axis values 1, 2, 3 and 4 denote the experiment set I, II, III and IV respectively. It

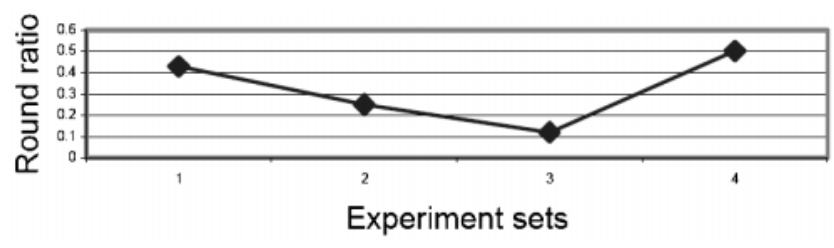

Fig. 8. Round ratio values for experiment I, II, III and IV.

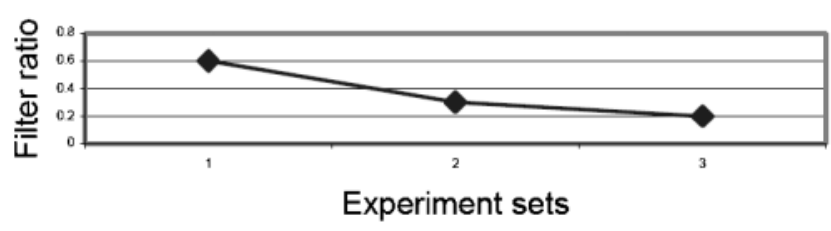

Fig. 9. Filter ratio values for experiment I, II, and III.

is evident from the Fig.8 and Fig.9 that both the round ratio and filter ratio decrease significantly with the increment of the size of SG. This indicates that the algorithm terminates in first round of election process most of the times when the size of SG increases. It is interesting to note that, the round ratios are $0.428,0.25$ and 0.12 for the first three sets of experiments where the sizes of SG are 5, 10 and 15 respectively. However, in case of large distributed system having 150 MDVM servers, the round ratio is 0.5 , which is significantly small for such a large distributed system. The filter ratio values of $0.6,0.3$ and 0.2 in experiments I, II and III indicate that the algorithm successfully restricts the number of servers entering in the second round election phase. In case of experiment IV, the filter ratio values vary in five experiment instances as shown in Fig.10. It is evident from Fig.10 that the maximum number of MDVM servers entering in second round election phase is 9 out of 150 (filter ratio $=0.06$ ), which is significantly small. The comparative study of round-trip latency of network-paging values is given in Fig.11. The algorithm is realizable and in the four experiment sets, the algorithm terminates successfully by electing a unique leader and co-leader.

\section{Conclusions}

In this paper the concept of MDVM system and the architecture of cellular network containing the ServerGroup (SG) are described. A randomized distributed algorithm is presented to elect a leader and co-leader of 


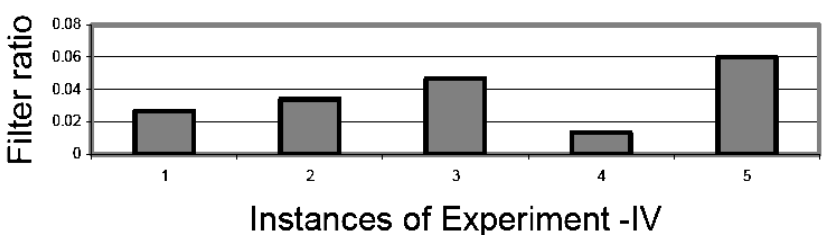

Fig. 10. Filter ratio values for experiment-IV.

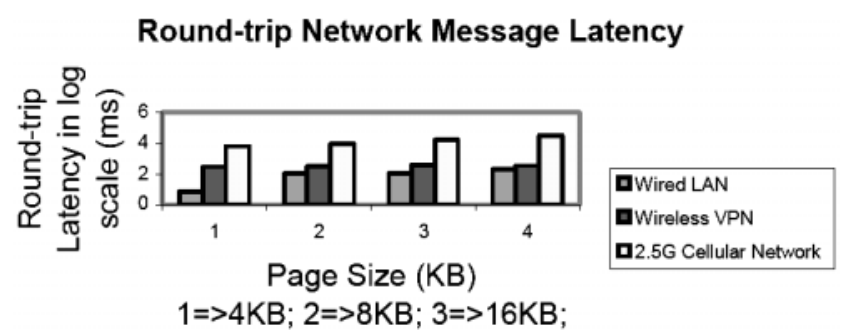

Fig. 11. Network-paging latency values.

SG and network-paging latency values are experimentally evaluated for LAN, Wireless VPN and 2.5G GPRS systems connecting a mobile client and a server in SG. The proposed algorithm assigns the node IDs to the MDVM servers online and does not rely on the static pre-assigned node IDs. The algorithm is implemented in the distributed system setup. The experimental results illustrate that the algorithm terminates in the first round of election phase in majority cases by electing a unique leader and co-leader of the SG. The round ratio values decrease with the increase in the number of MDVM servers. In addition, the number of randomly chosen servers entering in the second round of election phase is substantially reduced as compared to the initial set of servers. The experimental results illustrate that the filter ratio values decrease significantly with the increase in the size of SG. The proposed algorithm is realizable, free from any assumption regarding static node IDs, network topology and buffer space limitations. The experimental values of network-paging latencies between the mobile-clients and servers of the SG indicate that MDVM system comprised of SG is realizable using 3G/4G mobile communication technologies. The overall message complexity of the algorithm is $\mathrm{O}\left(\left|N_{a}\right|\right)$ where, $\left|N_{a}\right|$ is the total number of MDVM servers in a SG.

\section{References:}

[1] G. Antonoiu and P. K. Srimani "A Self-Stabilizing Leader Election Algorithm for Tree Graphs," In the Journal of Parallel and Distributed Computing, 34(2), pp. 227-232, 1996.

[2] S. Vasudevan, J. Kurose, and D. Towsley, "Design and Analysis of a Leader Election Algorithm for Mobile Ad-hoc Networks," In the proc. of $12^{\text {th }}$ IEEE International Conference on Network Protocols, 2004.

[3] S. Dulman et al., "Wave Leader Election Protocol for Wireless Sensor Networks," In proc. of $3^{\text {rd }}$ International Symposium on Mobile Multimedia Systems and Applications, pp. 43-50, 2002.

[4] S. Vasudevan, B. Decleene, I. Immerman, J. Kurose, and D. Towsley, "Leader Election Algorithms for Wireless Ad-hoc Networks," DARPA Information Survivability Conference and Exposition, Vol.I, IEEE CS Press, 2003.
[5] N. Malpani, J. Welch, and N. Vaidya, "Leader Election Algorithms for Mobile Ad-hoc Networks," In proc. of $4^{\text {th }}$ International Workshop on Discrete Algorithms and Methods for Mobile Computing and Communications, Boston, 2000.

[6] I. Gupta, R. Renesse, and K. Birman, "A Probabilistically Correct Leader Election Protocol for Large Groups," In proc. of International Symposium on Distributed Computing, Spain, 2000.

[7] G. Itkis, C. Lin, and J. Simon, "Deterministic, Constant Space, SelfStabilizing Leader Election on Uniform Rings," In proc. of $9^{\text {th }}$ International Workshop on Distributed Algorithms, Springer LNCS, 1995.

[8] G. Frederickson and N. Lynch, "The Impact of Synchronous Communication on the Problem of Electing a Leader in a Ring," $16^{\text {th }}$ Annual ACM Symposium on Theory of Computing, pp. 493-503, 1984.

[9] D. Ellie and P. Prakash, "Leader Election and Distributed Consensus with Quantum Resources," Tech. Rep., McGill University, Canada, 2005.

[10] G. Frederickson and N. Lynch, "Electing a Leader in a Synchronous Ring," In the Journal of ACM, Vol.34, No.1, pp. 98-115, 1987.

[11] B. Joffroy, G. Maria, and C. Johnen, "Randomized Self-Stabilizing and Space Optimal Leader Election under Arbitrary Scheduler on Rings," Research Report No.1225, L.R.I., http://www.lri.fr/ $/$ colette/ps/rap1225.ps, 1999

[12] H. Attiya and J. Welch, "Distributed Computing: Fundamentals, Simulations and Advanced Topics," London, UK, McGraw-Hill, 1998.

[13] G. Tel, "Introduction to Distributed Algorithms," 2 nd Edition, Cambridge University Press, 2000.

[14] N. Lynch, "Distributed Algorithms," Morgan Kaufmann Publishers, 1996.

[15] R. Gallager et al., "A Distributed Algorithm for Minimum Weight Spanning Trees," In ACM Transactions on Programming Languages and Systems, Vol.4, No.1, pp. 66-77, 1983.

[16] D. Peleg, "Time Optimal Leader Election in General Networks," In Journal of Parallel and Distributed Computing, Vol.8, No.1, pp. 96-99, 1990.

[17] J. Burns, "A Formal Model for Message Passing Systems," TR 91, Indiana University, USA, 1980.

[18] D. Hirschberg et al., "Decentralized Extrema-Finding in Circular Configurations of Processors," Communication ACM, Vol.23, No.11, pp. 627-628, 1980.

[19] B. Chor and C. Dwork, "Randomization in Byzantine Agreement," Advances in Computing Research, Vol.5, pp. 443-498, 1989.

[20] S. Bagchi, "Design Architecture and Model of MDVM System," In proc. of IFIP International Conference on Intelligence in Communication Systems, Springer-Verlag LNCS, Vol.3283, 2004.

[21] M. Nadia and Y. Kin, "Designing Wireless Enterprise Applications on Mobile Devices," ICITA 2002.

[22] MOWAHS, IDI, NTNU, http://www.mowahs.com/, 2003.

[23] E. Pitoura et al., "Dealing with Mobility: Issues and Research Challenges," TR-CSD-93-070, 1993.

[24] R. Badrinath et al., "Impact of Mobility on Distributed Computations," ACM OS Review, 1993.

[25] A. Black and J. Inouye, "System Support for Mobility," ACM SIGOPS, Ireland, 1996.

[26] E. W. Weisstein, "Random Number," From MathWorld-A Wolfram Web Resource, http://mathworld.wolfram.com/RandomNumber.html

[27] B. Schilit and D. Duchamp, "Adaptive Remote Paging for Mobile Computers," TR-CUCS-004-91, Columbia University, February, 1991.

[28] Y. Shigemori et al., "A proposal of a Memory Management Architecture for Mobile Computing Environment," IEEE DEXA, 2000. 


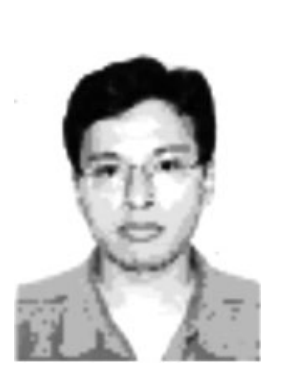

\section{Name:}

Susmit Bagchi

\section{Affiliation:}

Deptt. Of Information Technology, Sikkim Manipal Institute of Technology, India

\section{Address:}

Sikkim Manipal Institute of Technology, Majitar, Rangpo, East Sikkim, India

\section{Brief Biographical History:}

1993 Obtained B.Sc. (Hons.) from Calcutta University

1997 Obteined B.E. (Electronics Engg.) from Nagpur University

1999 M.E. (Electronics and Telecomm. Engg.) from Bengal Engineering and Science University

1999-2000 Scientist-B, DRDO, Ministry of Defence, India

2000-2005 Samsung Electronics Ltd., R\&D Centre, South Korea and

NTNU, Norway as research engineer and researcher respectively

2005-2006 Lecturer at Bengal Engineering and Science University

2006 Joined SMIT as Reader in the Department of Computer Engineering

Main Works:

- Operating Systems, Distributed Systems, Mobile Computing.

Membership in Learned Societies:

- AMIETE
Name:

Purnendu Das

Affiliation:

PD School of Information Technology, Bengal Engineering and Science University, India

Address:

Bengal Engineering and Science University, Shibpur, Howrah, India

Brief Biographical History:

1973 Obtained Ph.D. in Computer Science from Clarkson University, New York

2005 Professor at Bengal Engineering and Science University in the School of Information Technology (PDSIT)

Main Works:

- Object Technology, Programming Languages.

\section{Name:}

Hafizur Rahaman

\section{Affiliation:}

Deptt. Of Information Technology, Bengal Engineering and Science

University, India

Address:

Bengal Engineering and Science University, Shibpur, Howrah, India

Brief Biographical History:

1986 Obtained B.E. from Bengal Engineering and Science University

1988 Obtained M.E. from Jadavpur University

2003 Obtained Ph.D. from Jadavpur University

2005 Assistant Professor at Bengal Engineering and Science University in the department of Information Technology

\section{Main Works:}

- Logic Synthesis, Fault Tolerant Computing, System-on-Chip design and testing. 